\title{
Polarization Properties of Laser-Diode-Pumped Microchip Nd:YAG Ceramic Lasers
}

\author{
Kenju Otsuka \\ Tokai University \\ Japan
}

\section{Introduction}

Laser-diode(LD)-pumped solid-state lasers have attracted much attention as compact, highly efficient light sources for various applications. A wide variety of materials have been studied to develop more efficient and high power microchip lasers with LD pumping. In end-pumping schemes, materials with a short absorption length for the LD pump beam are most promising for highly efficient operations because of the excellent match between the mode and pump beam profiles. In the past decade, new single-crystalline laser materials having increased absorption coefficients for LDs, such as $\mathrm{Nd}: \mathrm{GdVO}_{4}$ and $\mathrm{Yb}: \mathrm{YAG}$, have been reported. On the other hand, the recent advancement of sintering methods led to the development of polycrystalline ceramic laser materials that have transparency comparable to single crystals (Ikesue et al., 1995 a, b). Various ceramic laser materials, e.g., host crystals and active ions, have been sintered toward LD-pumped solid-state lasers. Intensive studies are going on toward high power lasers utilizing such advantages of ceramic lasers as the growth of samples with large arbitrary shapes and high doping of active ions.

From laser physics point of view, on the other hand, ceramic lasers, which consist of randomly distributed single crystals surrounded by grain boundaries, are interesting active materials for studying lasing properties in random media. This Chapter is devoted to the formation of transverse modes and polarization characteristics in Nd:YAG ceramic lasers, focusing on the effect of average grain sizes on lasing properties.

\section{Formation of local transverse modes and associated dynamic effects}

Systematic studies of Nd:YAG ceramic lasers for different doping levels showed that if we dope $\mathrm{Nd}^{3+}$-ions highly, it is difficult to keep the large grain size and the number of grain boundaries will increase within the same path length accordingly (Ikesue et al., $1995 \mathrm{a}$, b; Shoji et al., 2000; Lu et al., 2000). In this section, our experimental results concerning lasing pattern formations due to grain boundaries and the resultant dynamic instabilities observed in Nd:YAG ceramic lasers (Kawai et al, 2004; Narita et al., 2005; Otsuka et al., 2006) are reviewed.

\subsection{Segregation into local transverse modes and dynamic instability}

Thin-slice Nd:YAG ceramic lasers consist of randomly distributed single-crystalline grains, whose directions of crystal axes surrounded by grain boundaries are independent, placed 
between closely-spaced reflective end surfaces. Since the thermal birefringence (i.e., depolarization) strongly depends on the direction of crystal axes of the grain and thus strong phase distortions occur over transverse directions with increasing the pump-power. Due to resultant random phase disturbances distributed inside the plane-parallel cavity, Hermite-Gauss modes cannot be formed and the lasing pattern is expected to be split into multiple localized transverse modes, namely "local modes" hereafter, having slightly different lasing frequencies resulting from slightly different optical cavity lengths, i.e., standing-wave conditions.

The experiment was carried out using mirror-coated thin-slice Nd:YAG ceramic lasers with various $\mathrm{Nd}$ concentrations sintered by Polytechno Corp. to clarify the formation of local modes, in which stable optical resonators are formed by the thermal lens effect due to LD pumping. The experimental setup is shown in Fig. 1(a). All the samples were $5 \mathrm{~mm}$ square and $1 \mathrm{~mm}$ in thickness, and end surfaces were coated by dielectric mirrors $\mathrm{M}_{1}(99.9 \%$ reflection at $1064 \mathrm{~nm}$ and high transmission at $808 \mathrm{~nm})$ and $\mathrm{M}_{2}(98 \%$ reflection at $1064 \mathrm{~nm})$. A nearly collimated elliptical-shape pump beam from the LD with 808-nm wavelength was passed through a pair of anamorphic prisms to transform the elliptical shape into circular one, and then it was focused onto the surface of $\mathrm{Nd}$ :YAG ceramic lasers by microscope objective lenses with different magnifications, i.e., $M=10 x$ (numerical aperture, $N . A=0.25$ ) and 20x (N.A. = 0.4). Example scanning electron microscope (SEM) images of the etched surfaces, indicating single-crystalline grain structures, are shown in Fig. 1(b), in which a pump-beam size is depicted. Grain sizes are decreased with increasing Nd concentrations.

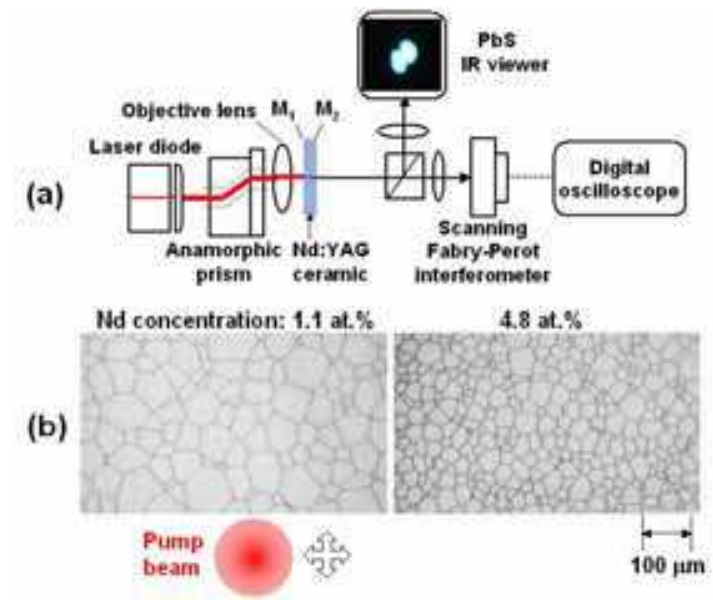

Fig. 1. (a) Experimental setup. (b) SEM image of the etched surfaces of Nd:YAG ceramic. The pump-beam spot size with $\mathrm{M}=10 \mathrm{x}$ and 20x focusing lens was about 80 and $40 \mu \mathrm{m}$.

In order to identify expected splits into spatially-distributed local modes, we measured near-field patterns, corresponding optical spectra and output waveforms. Lasing patterns and the corresponding detailed oscillation spectra were measured using the same $\mathrm{PbS}$ infrared viewer (Hamamatsu Photonics, C-1000) and scanning Fabry-Perot interferometer (Burleigh, SA Plus, free-spectral range: $2 \mathrm{GHz}$; resolution: $6.6 \mathrm{MHz}$ ). Here, a single longitudinal-mode operation in the entire pump-power region was confirmed by a multiwavelength-meter. Example results are shown in Figs. 2. A knife edge placed close to the 
laser was used to block a part of the lasing beam. Near-field patterns for the whole and partial beams and their optical spectra are shown in Figs. 2 (a)-(b). In this case, two local modes separated by $250 \mathrm{MHz}$ are oscillating at their own frequencies. A high-speed intensity modulation at $250 \mathrm{MHz}$ was observed as shown in a waveform of Fig. 2(c). The corresponding power spectrum peaks at $250 \mathrm{MHz}$ and its harmonic of $500 \mathrm{MHz}$. Therefore, the periodic waveform is not a simple beat note, but it arises from the intensity modulation at the beat frequency.

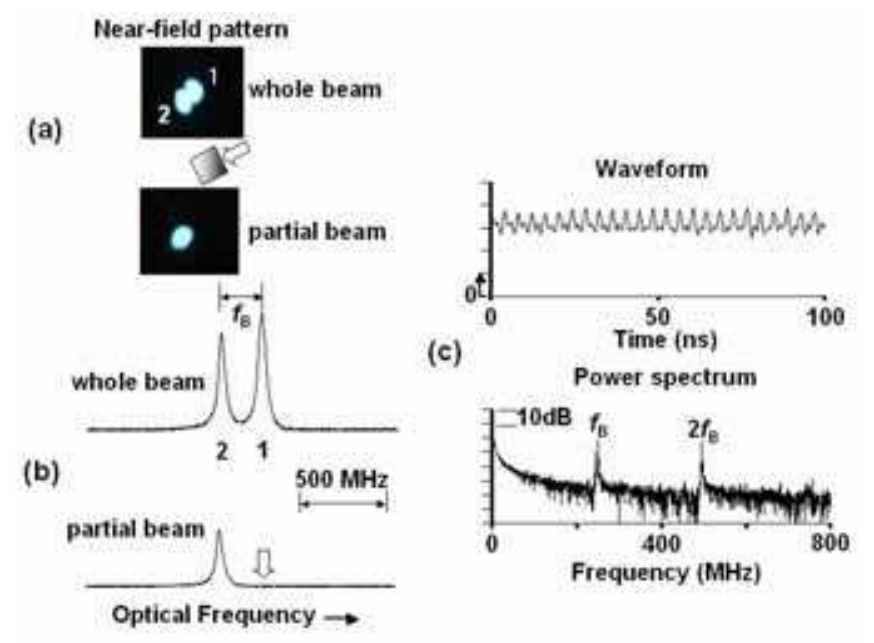

Fig. 2. (a) Near field patterns, (b) optical spectra, (c) intensity waveform and the corresponding power spectrum observed in the 1.1 at.\%-doped Nd:YAG ceramic laser.

Various modulation patterns featuring periodic and breathing-type waveforms at beating frequencies of adjacent local modes were observed by slight changes in pump positions. Furthermore, the modulation amplitude was found to be greatly enhanced by a fine tuning of the pump position with a larger magnification objective lens. A variety of high-speed modulation waveforms at frequencies up to $1 \mathrm{GHz}$, which is much higher than the relaxation oscillation frequency of few $\mathrm{MHz}$, was observed in the present experiment for slightly different pump positions, e.g., several tens of microns, at a fixed pump power. Example high-frequency modulation waveforms and corresponding power spectra observed for different pump-beam (i.e., lasing-beam) diameters observed in a 3.6 at.\% doped sample are shown in Fig. 3. Complicated waveforms modulated by many high frequency beats among local modes was observed for a larger pump-beam diameter [(a)], while larger amplitude breathing-type waveforms involving small number of local modes took place for a tightly focused pump-beam [(b)] in which the threshold pump power was $92 \mathrm{~mW}$ and the slope efficiency was $20 \%$. With increasing lasing beam diameter larger by the use of smaller magnification lenses, the number of grain boundaries increases across the lasing beam increases. As a result, the number of interacting local modes, i.e., beat frequencies, increases accordingly, yielding complicated modulation waveforms.

Quasi-periodic and chaotic modulation waveforms around at the relaxation oscillation frequency were also observed as shown in Figs. 4(a)-(b) at the same pump power as Fig. 3, where the pump beam was tightly focused using the $20 x$ (N.A. $=0.4$ ) magnification lens. 

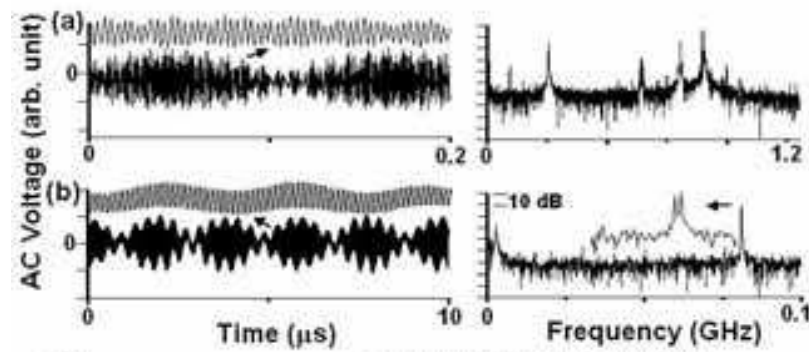

(c)

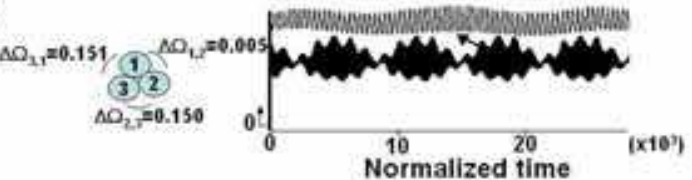

Fig. 3. Intensity waveforms and the corresponding power spectra. (a) High-speed multiplefrequency modulation, (b) Breathing-mode modulation. (c) Numerically reproduced breating-mode modulation.

The simultaneous measurement of optical spectra revealed that these instabilities were confirmed to take place when a beat-frequency among coupled local modes approached the relaxation oscillation frequency. Provided averaged grain size of $20 \mu \mathrm{m}$ shown in Fig. 1(a), which is smaller than the measured focusing beam diameter of $40 \mu \mathrm{m}$, an extremely sensitive change in observed waveforms against a pump position change, i.e., grain structures, can be well understood since a slight change of pump position could results in a change in the spatial arrangement of local modes within the lasing beam, e.g., nearest neighbors, having different lasing frequencies at which beating modulations take place.

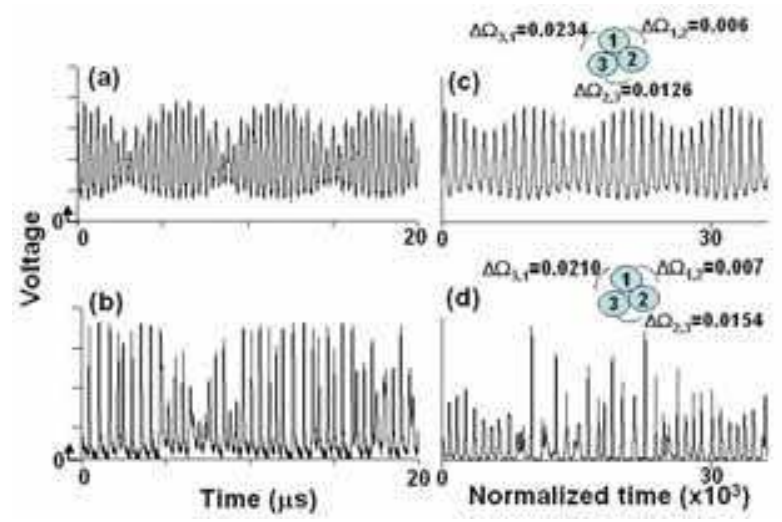

Fig. 4. Observed intensity waveforms (left) and numerical reproductions (right).

(a), (c): Quasi-periodic relaxation oscillations, (b), (d): Chaotic relaxation oscillations.

\subsection{Theoretical model}

Here, the theoretical reproduction of observed dynamic instabilities is shown based on the model of coupled local-mode lasers (Otsuka et al. 2006). 
The local modes are coupled through spatial field overlapping across the transverse direction. Assuming the nearest neighbor coupling among spatially-distributed local modes, the model equation of such coupled lasers are given as follows (Otsuka et al., 2000):

$$
\begin{gathered}
\mathrm{d} N_{\mathrm{i}} / \mathrm{d} t=\left\{w-1-N_{\mathrm{i}}-\left[1+2 N_{\mathrm{i}}\right] E_{\mathrm{i}}{ }^{2}\right\} / K, \\
\mathrm{~d} E_{\mathrm{i}} / \mathrm{d} t=N_{\mathrm{i}} E_{\mathrm{i}}+\eta_{\mathrm{i}, j} \Sigma E_{\mathrm{j}} \sin \Phi_{\mathrm{l}, j}, \\
\mathrm{~d} \Phi_{\mathrm{i}, \mathrm{j}} / \mathrm{d} t=\Delta \Omega_{\mathrm{i}, \mathrm{j}}-\eta_{\mathrm{i}, \mathrm{j}} \Sigma\left[\left(E_{\mathrm{j}} / E_{i}\right)+\left(E_{\mathrm{i}} / E_{\mathrm{j}}\right)\right] \sin \Phi_{\mathrm{l}, \mathrm{j},}
\end{gathered}
$$

Here, $E_{\mathrm{i}}=(g \tau)^{1 / 2} \mathrm{E}(\mathrm{t})$ is the normalized field amplitude of $i$-th local mode, $N_{\mathrm{i}}=g N_{\mathrm{th}} \tau_{\mathrm{p}}\left[N_{\mathrm{i}}(\mathrm{t})\right.$ $\left./ N_{\mathrm{th}}-1\right]$ is the normalized excess population inversion of $i$-th local mode where $N_{\text {th }}$ is the threshold population inversion. $g$ is the differential gain coefficient, where gain is defined as $G=G_{\text {th }}+\mathrm{g}\left(N_{\mathrm{i}}(\mathrm{t})-N_{\mathrm{th}}\right)$. $w=P / P_{\text {th }}$ is the relative pump power normalized by the threshold, $K=\kappa \tau$ is the fluorescence lifetime normalized by the damping rate of the optical cavity, $\kappa=\left(2 \tau_{\mathrm{p}}\right)^{-1}\left(\tau_{\mathrm{p}}\right.$ : photon lifetime), $\Phi_{1, j}$ is the phase difference between $i$-th local mode field and adjacent $j$-th local mode field, $\Delta \Omega_{\mathrm{i}, \mathrm{j}}=\left(\omega_{\mathrm{j}}-\omega_{1}\right) / \kappa$ is the normalized frequency difference between $i$-th local mode field and adjacent $j$-th local mode field. $\eta_{\mathrm{i}, \mathrm{j}}$ is the coupling coefficient between adjacent local mode fields, and $t$ is the time normalized by the damping rate of the optical cavity. In a weak coupling regime, $\Delta \Omega_{\mathrm{i}, \mathrm{j}}>>\eta_{\mathrm{i}, \mathrm{j}}$, phase-locking between local modes fails and beating modulations at $\Delta \Omega_{\mathrm{i}, \mathrm{j}}$ take place. Large $K$ values in thin-slice solid-state lasers with short photon lifetimes guarantee efficient high-speed modulation far above relaxation oscillation frequencies (Otsuka et al., 2002). Numerical high-speed breathing-type modulation, quasi-periodic and chaotic relaxation-oscillation waveforms, $\Sigma \mathrm{E}_{\mathrm{i}}{ }^{2}$, which correspond to Figs. 3(b), 4(a) and 4(b), are shown in Figs. 3(c), 4(c) and 4(d), respectively. Here, three coupled localized modes with $\Delta \Omega_{\mathrm{i}, \mathrm{j}}$ shown in figures are assumed.

\subsection{Periodic spiking operations}

Periodic spiking operations also appear with a precise tuning of the pump position. Such Qswitching type periodic spiking oscillations, which appeared even in the single-mode region near the lasing threshold, are shown to be brought about by a different dynamic origin from modal-beating mediate intensity modulations shown in Figs. 3-4. An example bifurcation diagram and typical waveforms obtained at a fixed pump position is shown in Fig. 5. The periodic spiking oscillation in the single-mode region PS [(b)] tended to coexist with highspeed beating modulations in two-mode region, featuring random switching between two dynamic states RS [(c)], and finally high-speed modulation dominated spiking oscillations as the pump power approached the threshold pump power in region $\mathbf{H S}[(\mathrm{d})]$. Note that the spiking oscillation frequency decreased with in decreasing the pump power.

Self-induced periodic "spiking" modulations observed for a tight pump-beam focusing condition have been explained theoretically assuming saturable absorber type of inclusions in grain boundaries. The standard single-mode laser rate equation including saturable absorbers are given by: (Erneux, 1990)

$$
\begin{gathered}
\mathrm{d} n_{\mathrm{l}} / \mathrm{d} t=\left(w_{1}-n_{\mathrm{l}}-n_{\mathrm{l}} s_{\mathrm{l}}\right) / K, \\
\mathrm{~d} n_{\mathrm{a}} / \mathrm{d} t=\left(w_{\mathrm{a}}-n_{\mathrm{a}}-\alpha n_{\mathrm{a}} s\right) / K \\
\mathrm{~d} s / \mathrm{d} t=2\left(n_{\mathrm{l}}-n_{\mathrm{a}}-1\right) s,
\end{gathered}
$$


where $n_{\mathrm{l}}, n_{\mathrm{a}}$ are normalized population densities for the laser and saturable absorber, respectively, $s$ is the photon density, $w_{1}, w_{\mathrm{a}}$ are normalized pump rates and $\alpha$ is the saturation parameter. Observed periodic spiking oscillations, which appeared near the lasing threshold $w=P / P_{\text {th }}=w_{1} /\left(w_{\mathrm{a}}+1\right)<2$, have been well explained by the linear stability analysis and numerical simulation of single-mode nonlinear equations described above. An example numerical result is shown in Fig. 6. It was found theoretically that the periodic spiking oscillation starts to appear just above the threshold pump power, i.e., $w=1$, and the pulsation frequency increases as the pump power increases. In the real experiment, however, such a pulsation sometimes coexists with beating-type high-speed modulations shown in Fig. 5(d). Observed spiking oscillations and theoretical reproductions strongly suggest the possible inclusion of saturable absorber-type defects into grain boundaries in highly-doped Nd:YAG ceramics.

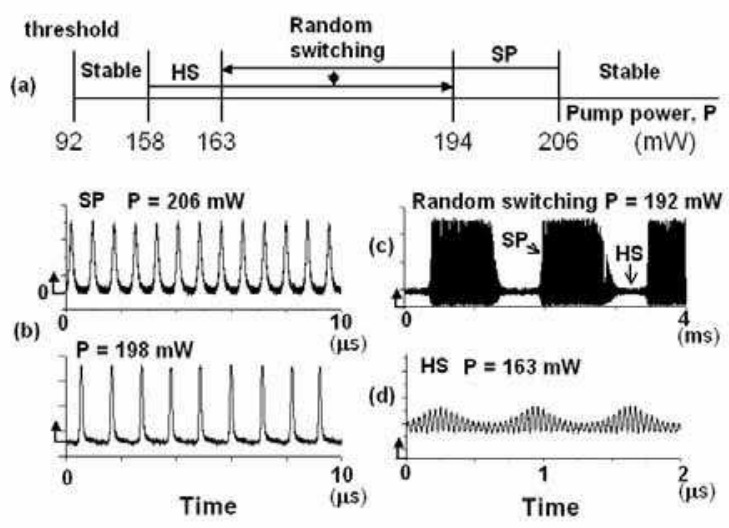

Fig. 5. (a) Bifurcation diagram as a function of the pump power observed at the fixed pump position. HS: high-speed modulation, SP. periodic spiking oscillation. (b) Observed Qswitching-type of spiking oscillation. (c) Observed random switching among SP and HS. (d) Observed high-speed modulation.
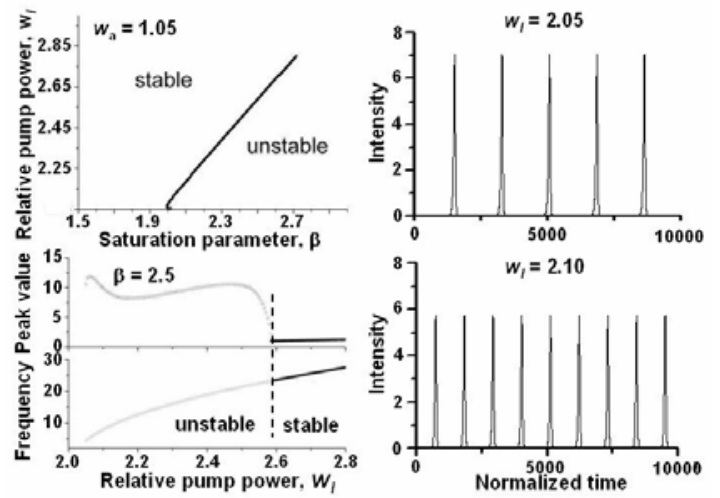

Fig. 6. Linear stability analysis and pulsation waveforms. 
Random switching behaviors among different dynamic states similar to Fig. 5(c) have been reported and interpreted in terms of chaotic itinerancy (Otsuka, 1999; Ko et al., 2001).

\subsection{Summary}

In this section, self-induced dynamic instabilities, featuring modal-beating mediate high speed modulations, large-amplitude quasi-periodic and chaotic relaxation oscillations, have been demonstrated experimentally using thin-slice Nd:YAG ceramic lasers with coated end mirrors. The theoretical model of coupled local-mode lasers including the effect of the lateral field overlapping, i.e., interference, among adjacent local modes has been presented for understanding dynamic instabilities. With a generalized model of coupled local-modes (i.e., filament) lasers proposed here, a variety of modal-beating mediate dynamic instabilities have been well reproduced. Self-induced Q-switching type of periodic spiking modulations observed for a tight pump-beam focusing condition have been also observed and explained theoretically assuming saturable-absorber type of inclusions in grain boundaries.

\section{Effect of average grain sizes on modal and polarization properties}

The inherent segregation of transverse patterns into multiple local modes possessing different lasing profiles occurs in thin-slice Nd:YAG ceramic lasers, whose grain sizes are several tens of micrometers as described in the previous section. On the other hand, singlefrequency linearly polarized emissions that were free from dynamic instabilities were achieved in microcrystalline Nd:YAG ceramic samples, whose average grain size was a few micrometers. In this section, we describe the effect of average grain size on transverse lasing patterns, detailed oscillation spectra, and polarization properties of LD-pumped Nd:YAG ceramic lasers (Ohtomo, 2007) to address the problems associated with making highly coherent miniature ceramic lasers possessing high beam quality and lasing frequency purity for applications besides high-power operation, e.g., laser metrology (Sudo et al. 2006).

\subsection{Input-output characteristics}

The experimental setup is shown in Fig. 7(a), in which an external cavity is used instead of mirror-coated thin-slice Fabry-Perot laser in the previous section to verify the effect of grain sizes on lasing pattern formations in the optical cavity, which should support a well-defined $\mathrm{TEM}_{00}$ mode. The experiment was carried out using two types of 1-mm-thick Nd:YAG ceramic sample having different average grain sizes, namely $\mathbf{A}$ and $\mathbf{B}$, sintered by Asaka Riken Co., Ltd. and Konoshima Chemical Co., Ltd., respectively, where Nd concentrations are 1.2 at.\% for $\mathbf{A}$ and 1.0 at.\% for $\mathbf{B}$. A linearly polarized collimated elliptical LD beam of $808 \mathrm{~nm}$ wavelength was transformed into a circular one and focused onto the ceramic samples using a microscope objective lens with a numerical aperture of 0.25 . The pumpbeam spot size averaged over the 1-mm-thick sample length was determined to be $80 \mu \mathrm{m}$, where the absorption coefficient for the LD light was 3.55 and $2.96 \mathrm{~cm}^{-1}$ for A and B samples, respectively. The sample was placed within a 5-mm-long semi-confocal cavity consisting of a flat mirror $\mathrm{M}_{1}(99.8 \%$ reflective at $1064 \mathrm{~nm}$ and $>95 \%$ transmissive at $808 \mathrm{~nm})$ and a concave mirror $\mathrm{M}_{2}(99 \%$ reflective at $1064 \mathrm{~nm})$ of $1-\mathrm{cm}$ radius of curvature. We have used several ceramic samples belonging to the same lot of A or B. Typical example SEM images of etched surfaces and histograms of grain sizes are shown in Fig. 8. 
Distinct differences in threshold pump powers and slope efficiencies were not found for both samples. The input-output characteristics of samples belonging to A were found to depend on samples and the pumped (i.e., lasing) beam position across the cross-sectional area of each sample, reflecting the grain structure within the lasing beam. While in samples belonging to B, such dependences were small. Typical examples are shown in Fig. 7(b).

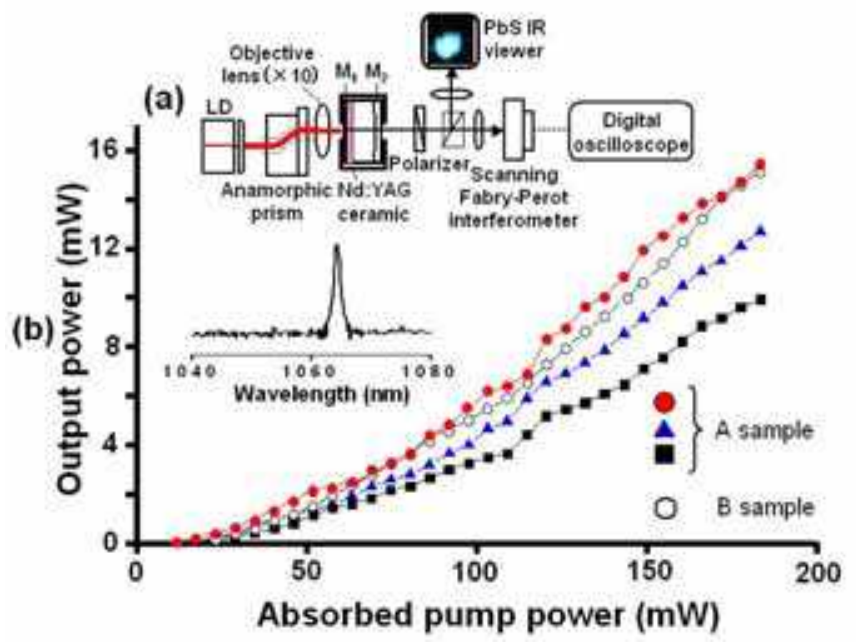

Fig. 7. (a) Experimental setup. (b) Input-output characteristics of large- and micro-grained ceramic lasers. A: large grain, B: micro-grain.
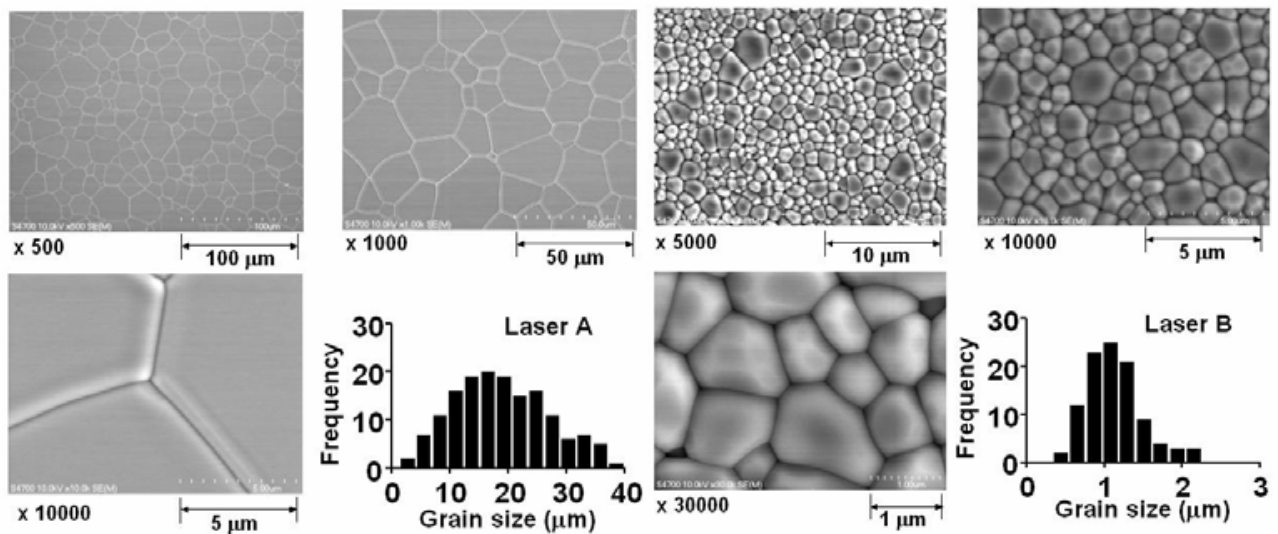

Fig. 8. SEM images of etched surfaces for several magnifications and measured histograms of grain sizes. Left: large grains, Right: micro-grains.

Larger undulations in output curves in samples A are considered to appear through structural changes of lasing patterns due to pump-dependent local-mode formations as will be discussed later. In all lasers, single-transition oscillations on the 1064-nm transition line were confirmed for the entire pump-power region by measurements using a multiwavelength meter (resolution: $0.1 \mathrm{~nm}$ ), as depicted in the inset of Fig. 7. This may result 
from the mode selection due to the etalon effect of the 1-mm-thick sample whose freespectral range of $\Delta \mathrm{f}=\mathrm{c} / 2 \mathrm{~nL}=56 \mathrm{GHz}$ (c: velocity of light, $\mathrm{n}=1.82$ : refractive index, $\mathrm{L}=1$ $\mathrm{mm}$ : sample length) is comparable to the gain bandwidth (full width at half maximum) of Nd:YAG ceramic.

\subsection{Lasing patterns and polarization states}

Lasing patterns and the corresponding detailed oscillation spectra were measured using the same PbS infrared viewer and scanning Fabry-Perot interferometer as those in Fig. 1 after the output beam had passed through a polarizer. Example results for the two lasers are shown in Fig. 9, where grain structures (i.e., SEM images of thermally etched surfaces), nearfield patterns and lasing optical spectra are also indicated.

The formation of local modes occurred in laser A just above the threshold pump power and the number of local modes increased with increasing pump power (i.e., transverse gain region). In the case of Fig. 9(a), a transverse mode belonging to the particular longitudinal mode was segregated into 4 local modes, possessing slightly different frequencies and polarization states. Note that the lasing beam consists of linearly polarized separated local modes with different polarization directions indicated by arrows. Laser A consists of singlecrystal grains of several tens of micrometers in size whose crystal axes are randomly distributed, while the spot size of the cavity eigenmode averaged over the sample length was calculated to be $80 \mu \mathrm{m}$, in which the average grain size was determined to be $19.22 \mu \mathrm{m}$ for the sample shown in Fig. 9(a). Therefore, several single-crystalline grains, whose sizes are orders of magnitude larger than the lasing wavelength, are included within a cavity eigenmode cross section on average, and the oscillation frequency and eigenpolarization state of the lasing beam cannot be uniquely determined because of position-dependent thermal birefringence across the beam. It thus appears that the lasing transverse mode should be split into local modes having different frequencies and polarization states, exhibiting dynamic instabilities as described in section $\mathbf{2}$.

(a)

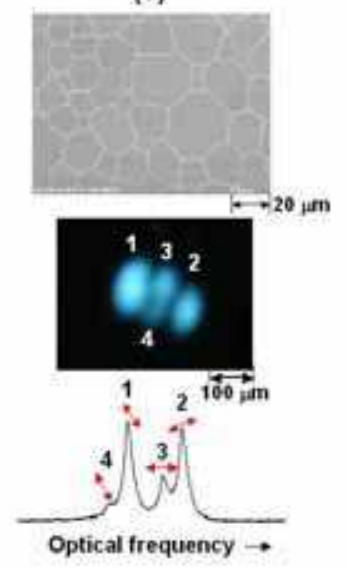

(b)

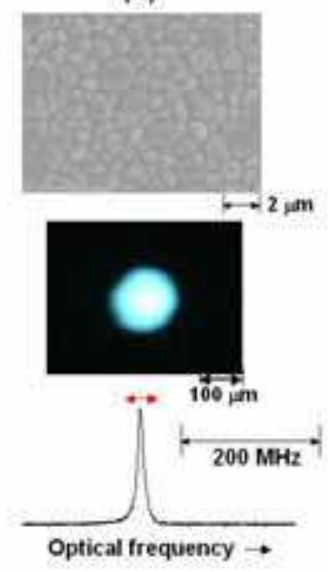

Fig. 9. SEM images of etched Nd:YAG ceramic surfaces, near-field patterns and optical spectra. (a) Large grain sample A, (b) micro-grained sample B. Polarization directions are indicated by red arrows. 
In the case of laser B, on the other hand, linearly polarized oscillations were obtained in a single $\mathrm{TEM}_{00}$ transverse mode (i.e., Hermite-Gauss $\mathrm{HG}_{00}$ mode), as shown in Fig. 9 (b). In this case, single-crystalline grain sizes are on the order of the lasing wavelength [i.e., average grain size of $1.16 \mu \mathrm{m}$ for the sample shown in Fig. 9(b)], and local modes cannot be formed within the eigenmode (i.e., TEM $_{00}$ mode) spot size. Consequently, segregations into local modes can be substantially suppressed, leading to stable single-mode operations without dynamic instabilities. An example far-field intensity profile of laser B is shown in Fig. 10, together with a Gaussian fitting curve, where a laser-beam profiler (Coherent, Beam Master3Si) was used. The mode purity, which is the degree to which the intensity pattern reproduces the theoretical $\mathrm{TEM}_{00}$ mode (Arlt, 1998) was evaluated to be greater than $99 \%$ for the entire pump-power region.

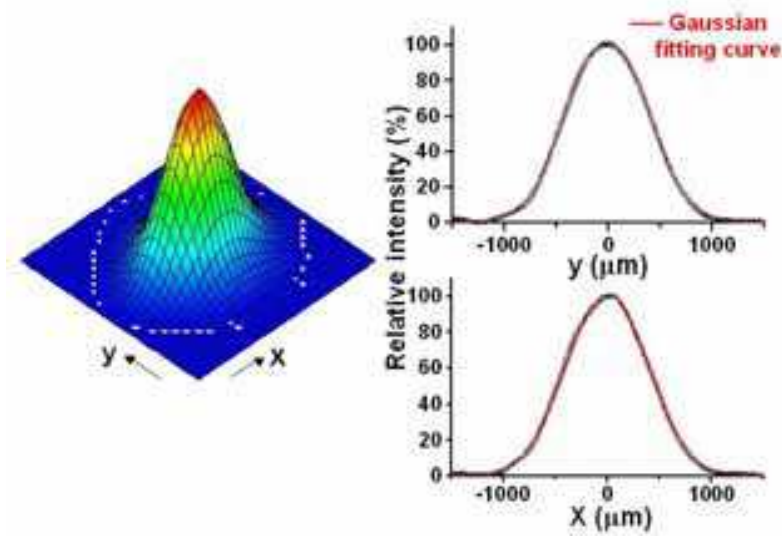

Fig. 10. Typical far-field lasing pattern of the micro-grained Nd:YAG ceramic laser.

Example pump-dependent optical spectra for other ceramic samples are shown in Fig. 11. Linearly polarized single-frequency oscillations, whose polarization direction coincided with that of the LD pump light, were obtained in laser B. In the high pump-power regime, the orthogonal linearly-polarized weak mode appeared as indicated by the double downarrow in this sample, featuring weak dual-polarization oscillations.

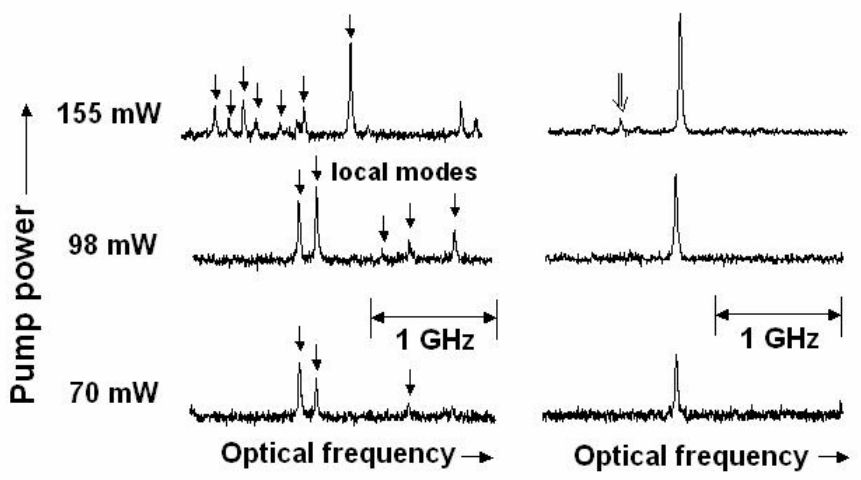

Fig. 11. Pump-dependent optical spectra. Left: large grains, Right: micro-grains 
We tested 40 samples including Yb:YAG with different average grain sizes purchased from different suppliers and observed the formation of local modes in all the samples except for micro-grained samples with average grain sizes smaller than approximately $5 \mu \mathrm{m}$. The results are summarized in Table I. Stable linearly-polarized TEM ${ }_{00}$ mode operations without dynamic instabilities were observed in a mirror-coated 5 at.\%-doped 2-mm-thick Yb:YAG micro-grained ceramic laser with average grain sizes of $3.2 \mu \mathrm{m}$ (Ohtomo, 2007) and its application to the self-mixing laser metrology with enhanced optical sensitivity has been demonstrated (Ohtomo, 2009).

\begin{tabular}{|c|c|c|c|c|c|c|c|}
\hline $\begin{array}{c}\text { Doping } \\
(\text { at. \%) }\end{array}$ & $\begin{array}{c}1.1 \\
(\mathbf{N d})\end{array}$ & $\begin{array}{c}2.4 \\
(\mathbf{N d})\end{array}$ & $\begin{array}{c}4.8 \\
(\mathbf{N d})\end{array}$ & $\begin{array}{c}1.2 \\
(\mathbf{N d})\end{array}$ & $\begin{array}{c}4.8 \\
(\mathbf{N d})\end{array}$ & $\begin{array}{c}5.0 \\
(\mathbf{Y b})\end{array}$ & $\begin{array}{c}1.0 \\
(\mathbf{N d})\end{array}$ \\
\hline $\begin{array}{c}\text { Grain size } \\
(\mu \mathrm{m})\end{array}$ & $\mathbf{5 1 . 8 5}$ & $\mathbf{3 7 . 3 5}$ & $\mathbf{1 9 . 0 3}$ & 19.22 & 5.61 & 3.20 & 1.16 \\
\hline Lasing mode & LMs & LMs & LMs & LMs & LMs & TEM $_{00}$ & TEM $_{00}$ \\
\hline
\end{tabular}

Table I. Average grain size vs modal structure observed in various ceramic YAG samples purchased from different suppliers. LMs: local modes. The average grain size was determined by averaging the length along the longest direction of each grain.

\subsection{Discussions}

Let us discuss the polarization properties observed in LD-pumped miniature ceramic lasers, in comparison with those in single-crystalline lasers. In LD-pumped thin-slice singlecrystalline Nd:YAG lasers, we observed linear-polarization oscillations in the low pumppower region, and dual-polarization oscillations (DPO) appeared in the wide pump-power region. A typical input-output characteristic observed in a 3-mm-diameter, 5-mm-thick mirror-coated Nd:YAG single-crystalline laser with LD pumping is shown in Fig. 12. Here, mirror coatings are the same as those shown in Fig. 7(a). The threshold pump power is larger than those of ceramic lasers shown in Fig. 7(b) because of the increased lasing beam spot size of $200 \mu \mathrm{m}$ (i.e., mode volume). In a wide pump-power region, DPO is found to occur. The nonlinear complementary output characteristic was theoretically reproduced by the cross saturation of population inversion among orthogonally polarized modes (Otsuka et al., 2007). It was found that such polarization characteristics critically depend on the slight asymmetry in pumping geometry, i.e., a slight change in pump position with respect to the central axis of the Nd:YAG crystal. Such pump-dependent polarization has also been reported by Cabrera et al., 2005.

The intriguing question is why do linear or dual-polarization emissions exceed the unpolarized emissions expected in single-crystalline Nd:YAG with the isotropic fluorescence properties. A plausible physical origin might be attributed to the thermal birefringence inherent in Nd:YAG crystals, in which depolarization effect is minimum along the orthogonal directions across the center of the pump (i.e., lasing) beam stemming from the so-called four-leaf rose depolarization pattern (Koechner, 1970; Otsuka, 1971) as depicted in the left of Fig. 12. In short, in LD-pumped miniature laser configurations with small pump-beam (i.e., lasing) spot sizes like the present lasers, the central region of the lasing beam does not suffer from the depolarization effect in the case of orthogonal linearly polarized emissions. Therefore, polarization eigendirections are imposed by such a thermally induced crystal birefringence, and DPOs are considered to exceed unpolarized emissions, as expected in the case of small gain anisotropy. 

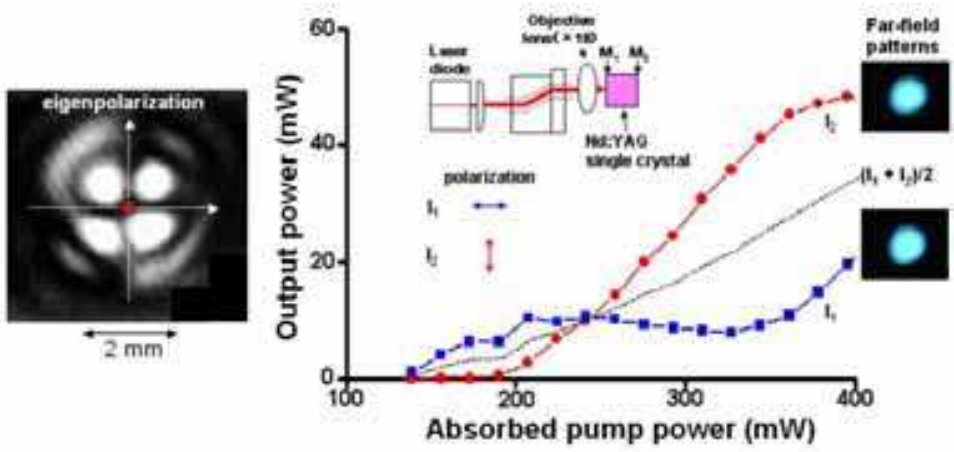

Fig. 12. Dual-polarization oscillation in the Nd:YAG single crystalline laser.

In micro-grained ceramic lasers, on the other hand, the averaging of thermal birefringence effect in Nd:YAG ceramics due to random distributions of crystal axes of grains reported by Shoji et al., 2002 is considered to become significant because of the extremely small grain sizes comparable to the lasing wavelength. Furthermore, in general, the thermally induced internal strain can be relaxed at grain boundaries in ceramics consisting of grains whose crystal axes are randomly distributed. With decreasing grain size, the suppression of thermal birefringence is expected to be more pronounced owing to increased grain boundaries. Indeed, depolarization patterns were observed to be substantially smoothed out in micro-grained ceramics. Therefore, micro-grained ceramic lasers are considered to act like highly isotropic lasers, being free from the thermal birefringence effect. Note that linearly polarized emissions predominate and DPO appears with increasing pump power in lowpower LD end-pumped Nd-doped fiber lasers in the absence of thermal birefringence (Bielawski, 1992). Such a lasing polarization property has been well explained theoretically in terms of the polarization-selective gain, namely pumping anisotropy, in which the polarization-selective gain occurs because both the interactions between the polarized pump and the ions, and that between the ion and the fields along each direction of polarization depend on the local field experienced by the ion (Bielawski, 1992; Kravtsov et al., 2004). The observed polarization properties, which follow the pump polarization, in micro-grained ceramic lasers could be interpreted in terms of the pumping anisotropy in isotropic lasing media. This topic will be discussed later again in $\mathbf{4 . 2}$.

\section{Controlling modal and polarization properties}

This section is devoted to the control of transverse lasing patterns and their polarization properties of Nd:YAG ceramic lasers by physical manipulations toward stable singlefrequency linearly polarized emissions for various applications besides high-power applications.

\subsection{Linearly-polarized Ince-Gauss mode operations with off-axis LD pumping}

Here, let me show the unique method to prevent local-mode oscillations and achieve stable operations without dynamic instabilities, which has been realized by adjusting azimuthal 
cavity symmetry, in which forced Ince-Gauss (IG) mode operations take place in linearlypolarized single-frequency oscillations (Otsuka, 2007).

The experimental setup is the same as Fig. 7(a), where the two mirrors and the 1-mm-thick Nd:YAG ceramic sample of 1.2 at.\% were assembled into one body. In the present experiment, we used larger grain samples categorized into $\mathbf{A}$ in the previous section sintered by Asaka Riken Co., Ltd. which exhibited segregations into local transverse modes and associate dynamic instabilities in the usual LD pumping scheme, i.e., on-axis pumping. Far-field patterns and the corresponding detailed oscillation spectra were measured with the PbS infrared viewer and the scanning Fabry-Perot interferometer (free-spectral range: 2 $\mathrm{GHz}$, resolution: $6.6 \mathrm{MHz}$ ), after the output beam had passed through a polarizer similar to Fig. 7(a). Examples of the results for different rotation angles of the polarizer obtained in the low pump-power region are shown in Fig. 13. It is obvious that a transverse mode belonging to the particular longitudinal mode was segregated into closely-spaced three local modes, $\mathbf{a}$, $\mathbf{b}$, and $\mathbf{c}$, possessing slightly different frequencies and polarization states.

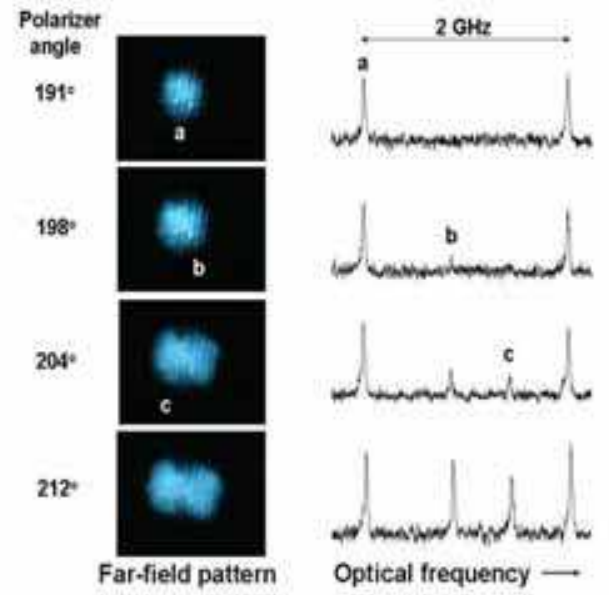

Fig. 13. Polarization-dependent far-field pattern change and the corresponding optical spectra.

As the pump power was increased, the number of local modes tended to increase due to the increase in active area in the transverse direction. The total and polarization-dependent farfield patterns are shown in Fig. 14, where polarization directions of individual local modes are indicated by arrows. Note that the lasing beam consists of linearly polarized separated 'clusters' of local modes with different polarization directions in this case. Dynamic instabilities resulting from beating-type modulations through field coupling among local modes described in section $\mathbf{2}$ were also observed in this particular sample.

Now let me show a simple method to achieve stable operations of Nd:YAG ceramic lasers to prevent segregations into local modes possessing different frequencies and polarization states by selective excitations of higher-order Ince-Gauss modes, whose mode volume can be larger enough than the grain size, with a controlled azimuthal cavity symmetry. Besides well-known Hermite-Gauss modes (HGMs) and Laguerre-Gauss modes (LGMs), Ince-Gauss modes (IGMs) is the third complete family of transverse eigenmodes. Ince-Gauss modes (IGMs) has been predicted as orthogonal solutions of the paraxial wave equation in elliptic 


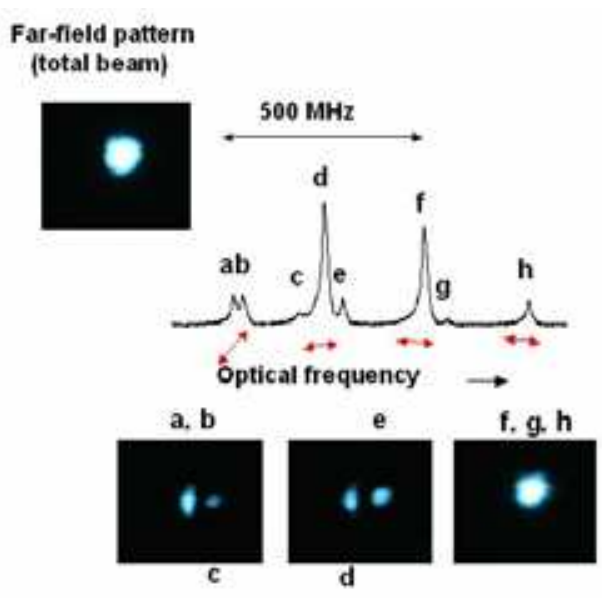

Fig. 14. Far-field patterns of total and partial beams, and their polarization states observed in the high pump-power regime.

coordinates and the IGMs with mode numbers $p$ and $m$, and ellipticity $\varepsilon$ are given by (M. Bandres \& Gutierrez-Vega, 2004).

$$
\begin{aligned}
& I G \mathrm{e}_{p, m}(\boldsymbol{r}, \varepsilon)=C\left[w_{0} / w(z)\right] C_{p}{ }^{m}(\mathrm{i} \xi, \varepsilon) C_{p}{ }^{m}(\xi, \varepsilon) \exp \left[-r^{2} / w^{2}(z)\right] \\
& \times \exp i\left[k z+\left\{k r^{2} / 2 R(\mathrm{z})\right\}-(\mathrm{p}+1) \psi_{z}(z)\right], \\
& I G_{p, m}^{\mathrm{o}}(\boldsymbol{r}, \varepsilon)=S\left[w_{0} / w(z)\right] S_{p}{ }^{m}(\mathrm{i} \xi, \varepsilon) S_{p}{ }^{m}(\xi, \varepsilon) \exp \left[-r^{2} / w^{2}(z)\right] \\
& \times \exp i\left[k z+\left\{k r^{2} / 2 R(\mathrm{z})\right\}-(\mathrm{p}+1) \psi_{z}(z)\right],
\end{aligned}
$$

where the elliptic coordinate is defined in a transverse $\mathrm{z}$ plane as $\mathrm{x}=f(\mathrm{z}) \cos \xi \cos \eta, \mathrm{y}=f(\mathrm{z})$ $\sin \xi \sin \eta$ and $\xi \in[0, \infty], \eta \in[0,2 \pi] . f(z)$ is the semifocal separation of IGMs defined as the Gaussian beam width, i.e., $f(z)=f_{0} w(z) / w_{0}$, where $f_{0}$ and $w_{0}$ are the semifocal separation and beam width at the $\mathrm{z}=0$ plane, respectively. $w(\mathrm{z})=w_{0}\left(1+z^{2} / z_{R}^{2}\right)^{1 / 2}$ describes the beam width, $z_{R}=k w_{0}^{2} / 2$ is the Rayleigh length, and $k$ is the wave number of the beam. The terms $C$ and $S$ are normalization constants, and subscripts $e$ and $o$ refer to even and odd IGMs, respectively. $C_{p}{ }^{m}(., \varepsilon)$ and $S_{p} m(., \varepsilon)$ are the even and odd Ince polynomials of order $p$, degree $m$, and ellipticity parameter $\varepsilon$, respectively. In Eqs. (7) and (8), $r$ is the radial distance from the central axis of the cavity, $R(\mathrm{z})=z+z_{R}{ }^{2} / z$ is the radius of curvature of the phase front, and $\Psi_{\mathrm{z}}(\mathrm{z})=\arctan \left(z / z_{R}\right)$. The parameters of ellipticity $\varepsilon$, waist $w_{0}$ and the semifocal seperation $f_{0}$ are not independent, but related by $\varepsilon=f_{0}{ }^{2} / w_{0}{ }^{2}$.

By adjusting azimuthal symmetry, a variety of higher-order IG mode oscillations, $\mathrm{IG}_{p, m}$, were selectively excited, where the central axis of the resonator was tilted against the pumpbeam axis as shown in Fig. 15(a) in the range of $0<\theta<30$ [mrad]. Such a tilt of the laser resonator assembled in one body against the pump-beam axis is considered to introduce an equivalent effect of the off-axis pumping (Schwarz et al., 2004) whose lateral shift ranges 0 $150 \mu \mathrm{m}$ from the center of the cavity. Example far-field lasing patterns observed for different azimuthal symmetries at a constant pump power of $293 \mathrm{~mW}$ are shown in Fig. 15(b), in 
which each output power was decreased to $5-7 \mathrm{~mW}$ from the output power of $10 \mathrm{~mW}$ in local-mode operations with on-axis pumping. The formation of IG modes by adjusting the LD pump-beam focus to the brightest 'target' spot of IGM lasing patterns, i.e., off-axis pumping, has been demonstrated by the numerical simulation (Chu \& Otsuka, 2007).

As for forced single IG mode oscillations, linearly-polarized single-frequency operations being free from dynamic instabilities have been achieved successfully, although the threshold pump power was increased to $80-100 \mathrm{~mW}$ depending on the tilt, i.e., astigmatic pumping, while higher-order HG modes were formed by a larger astigmatic pumping with further increased pump power. Linearly-polarized single-frequency operations were confirmed by optical spectra as shown in Fig. 15.

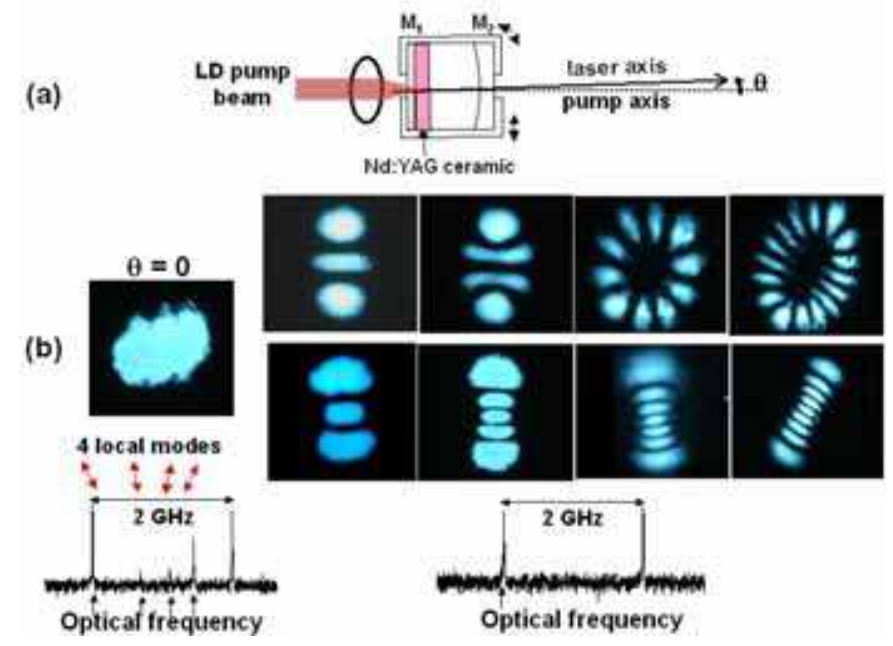

Fig. 15. (a) Azimuth laser-diode pumping scheme. (b) Far-field lasing patterns and the corresponding optical spectra. Left: On-axis pumping $(\theta=0)$; Right: off-axis pumping.

We have tested various 1-mm-thick Nd:YAG ceramic samples with 1.2 at. \%, 2.4 at. \% and 3.6 at.\% $\mathrm{Nd}$ concentrations in the same cavity configuration and forced single-frequency linearly-polarized IG mode operations were attained by adjusting azimuthal symmetry of the cavity against the LD-beam pump direction. Moreover, it is interesting to note that each single-frequency IG mode formed in the low pump-power region was maintained with increasing the pump power.

Single-mode IG patterns were formed stably in microchip Nd:YAG ceramic lasers with LD pump levels below $1 \mathrm{~W}$ presumably due to the less thermal lens effect. Analytical IG mode patterns with mode numbers $[p, m]$ and ellipticities $\varepsilon$ of the modes corresponding to observed patterns in Fig. 15 are shown in Fig. 16.

The mechanism here is that split of the fundamental TEM $\mathrm{T}_{00}$ mode into local modes due to grain boundaries under the mode-matched pumping condition is suppressed by the selective excitation of higher-order IG modes whose mode volumes are much larger than the averaged grain size under astigmatic pump. In other words, distributed thermal birefringence effects are effectively smoothed out in IG mode operations, resulting in singlefrequency linearly-polarized emissions similar to micro-grained $\mathrm{Nd}$ :YAG ceramic lasers with on-axis LD pumping demonstrated in section $\mathbf{3}$. 


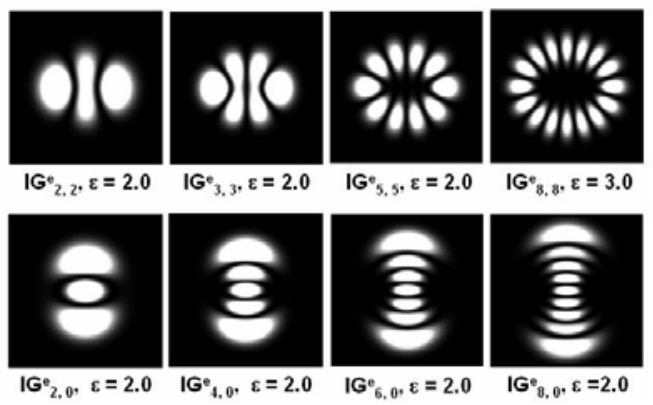

Fig. 16. Numerically reproduced IG mode patterns corresponding to observed IG mode patterns shown in Fig. 15(b).

\subsection{Observation of Mathieu-Gauss mode operations in micro-grained ceramic lasers} In this section, the lasing pattern formation in micro-grained Nd:YAG ceramic lasers with azimuth LD pumping is discussed. Spontaneous Mathieu-Gauss mode oscillations are shown to appear instead of IG mode operations, which take place in larger grained Nd:YAG microchip ceramic lasers with azimuth laser diode pumping (Tokunaga et al., 2009). Here, non-diffractive beams (Durin, 1987), including Bessel-Gauss (BG) (Arlt \& Dholakia, 2000) and Mathieu-Gauss (MG) modes (Gutierrez-Vega \& Bandres, 2007), are promising for sophisticated micromachining and for trapping microparticles and manipulating them over longer distances than any other optical tweezers. The effect of fluorescence anisotropy or thermal birefringence on these pattern formations is also discussed for several laser materials.

\section{A. Mathieu-Gauss and Bessel-Gauss mode operation}

The laser cavity configuration is the same as Fig. 7(a) and the 1-mm-thick micro-grained $\mathrm{Nd}$ :YAG ceramic of 1 at. $\% \mathrm{Nd}$ concentration was attached to $\mathrm{M}_{1}$ and the cavity was assembled into one body as depicted in Fig. 17(a). The averaged grain size was measured to

(a)

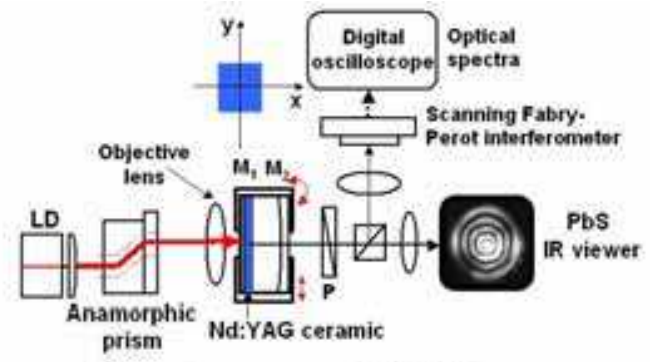
prism

Large-grain

(b)
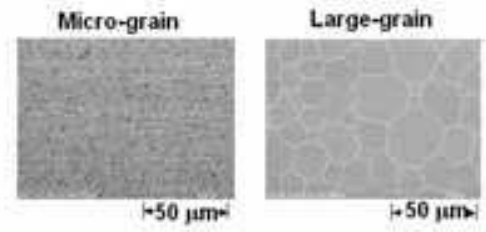

Fig. 17. (a) Experimental setup of micro-grained Nd:YAG ceramic lasers with azimuth LD pumping. (b) SEM images of etched surfaces. 
be $1.1 \mu \mathrm{m}$. An SEM surface image of the micro-grained sample used in this experiment is shown in Fig. 17(b), together with that of large-grain sample. The collimated linearlypolarized LD beam was passed through an anamorphic prism pair and it was focused onto the sample by a microscope objective lens of NA $=0.25$, where the focused beam diameter was about $80 \mu \mathrm{m}$. The laser exhibited a single-frequency TEM 00 -mode oscillation, which is linearly polarized along the LD pump-beam polarization direction due to the reduced thermal birefringence for mode-matched on-axis pumping condition as mentioned in section 3. By shifting or tilting the laser cavity slightly as depicted by arrows in Fig. 17(a), a variety of MG mode operations were observed, instead of Ince-Gauss (IG) modes, depending on the degree of effective off-axis pumping. Typical far-field patterns, including $\mathrm{BG}$ modes, are shown in Fig. 18. For the higher-order $\mathrm{BG}$ modes $\left(\mathrm{BG}_{1}, \mathrm{BG}_{2}\right)$, an optical vortex having a topological charge of 1 and 2 was formed in the center.

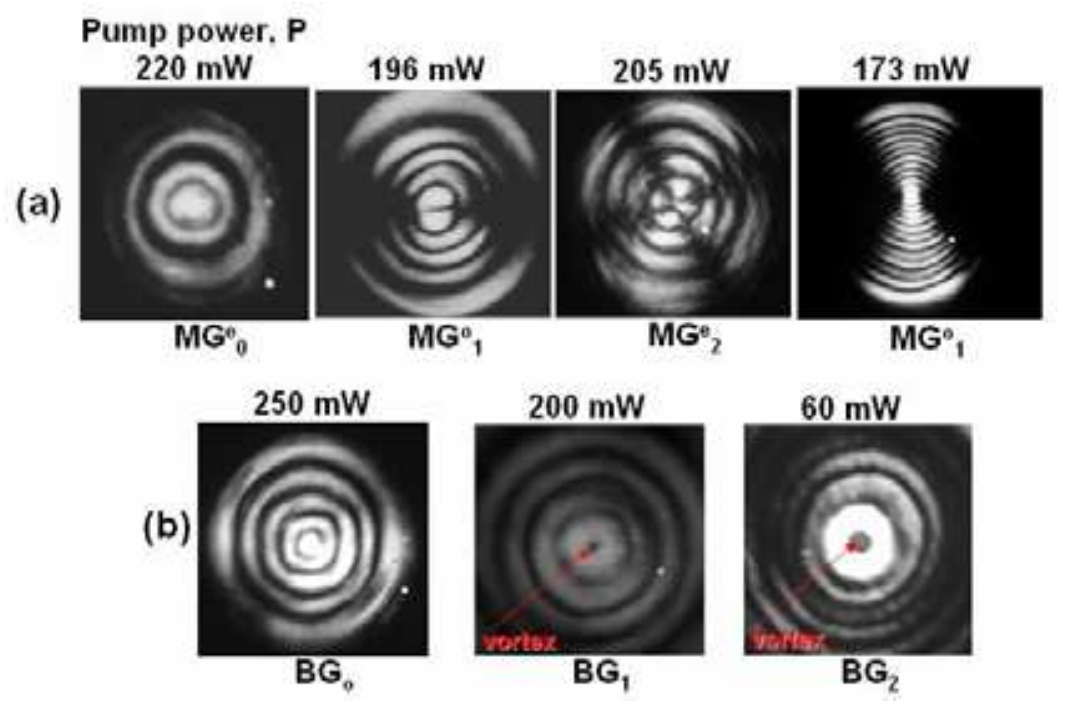

Fig. 18. Observed far-field lasing patterns. (a) Mathieu-Gauss laser beam. (b) Bessel-Gauss laser beam.

Numerically reproduced intensity patterns corresponding to Fig. 18 and the phase portraits are shown in Fig. 19. Here, the complex amplitude of the $m$-th order even and odd MG beams propagating along the positive $z$ of an elliptic coordinate system $\boldsymbol{r}=(\xi, \eta, z)$ is given by (Gutierrez-Vega \& Bandres, 2007):

$$
\begin{gathered}
M G^{e}{ }_{m}(r)=\exp \left(-\frac{k_{t}^{2} z}{2 k \mu}\right) G B(r) J e_{m}(\xi, q) c e_{m}(\eta, q), \\
M G^{o}{ }_{m}(r)=\exp \left(-\frac{k_{t}^{2} z}{2 k \mu}\right) G B(r) J o_{m}(\xi, q) s e_{m}(\eta, q) .
\end{gathered}
$$

Here, $J e_{m}(\cdot)$ and $J o_{m}(\cdot)$ are the $m$-th order even and odd radial Mathieu functions, $c e_{m}(\cdot)$ and $s e_{m}(\cdot)$ are the $m$-th order even and odd angular Mathieu functions, $G B(\boldsymbol{r})=\mu^{-1} \exp \left(-r^{2} / \mu w_{0}^{2}\right)$ is 
the fundamental Gaussian beam, $\mu(z)=1+\mathrm{i} z /\left(k w_{0}^{2}\right), w_{0}$ is the Gaussian width at the waist plane $z=0$, and $k=2 \pi / \lambda$ is the longitudinal wave number. $q=k_{t}^{2} f_{0}{ }^{2} / 4$ is the ellipticity parameter, which carries information about the transverse wave number $k_{t}$ and the semiconfocal separation at the waist plane $f_{0}$.

Similarly, $m$-th order BG beams are given by

$$
B G_{m}(r, \phi)=\exp \left(-\frac{k_{t}{ }^{2} z}{2 k \mu}\right) G B(r) J_{m}\left(\frac{k_{t} r}{\mu}\right) \exp (-i m \phi) .
$$

Here, $(\mathrm{r}, \phi)$ are the polar coordinates and $J_{m}(\cdot)$ is the $m$-th order Bessel function of the first kind.

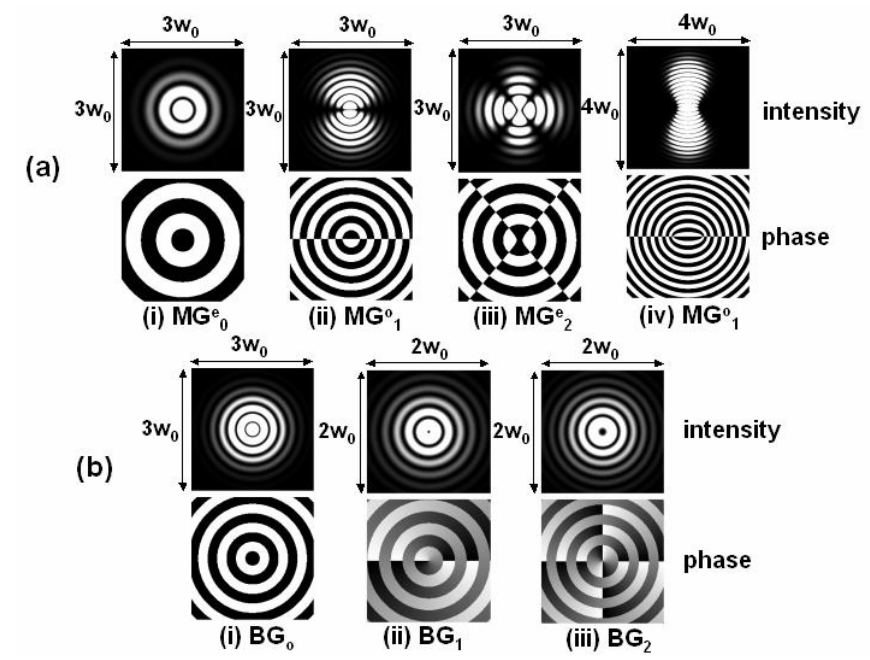

Fig. 19. Numerically reproduced intensity patterns corresponding to Fig. 18 and their phase portraits. (a) Mathieu-Gauss laser beam. (b) Bessel-Gauss laser beam. $\lambda$ (wavelength) $=1064$ $\mathrm{nm}, w_{0}=3 \mathrm{~mm}$. Adopted parameter values $\left(k_{\mathrm{t}}, q\right)$ are $(\mathrm{a})-(\mathrm{i}):(2800 / \mathrm{m}, 0.2) ;(\mathrm{a})-(\mathrm{ii}):(6000 / \mathrm{m}$, 0.2); (a)-(iii): (4300/m, 0.5); (a)-(iv): (7500/m, 25); (b)-(i): (4500/m, 0); (b)-(ii): (5500/m, 0); (b)(iii): $(6500 / \mathrm{m}, 0)$.

Elliptical-polarization BG modes or dual-polarization MG modes appeared for small effective off-axis pumping. An example of polarization-dependent oscillation spectra is shown in Fig. 20(a). With larger off-axis pumping, linearly polarized single or double longitudinal MG mode operations were observed, where the longitudinal mode spacing coincided with $12.88 \mathrm{GHz}$, which corresponds to the inverse of two round-trip times as expected for BG and MG mode oscillations. An example oscillation spectrum consisting of two longitudinal modes is shown in Fig. 20(b).

\section{B. Effect of fluorescence anisotropy on lasing pattern formation}

We replaced the micro-grained Nd:YAG ceramic by $\mathrm{LiNdP}_{4} \mathrm{O}_{12}(\mathrm{LNP})$ and a-cut Nd:GdVO crystals, which exhibit linearly polarized emission resulting from strong fluorescence anisotropy independently of the pump-beam polarization state. Under the same azimuth LD-pumping conditions as for micro-grained ceramic lasers, neither BG nor MG mode oscillations appeared. Instead, single-frequency linearly polarized IG mode operations on 


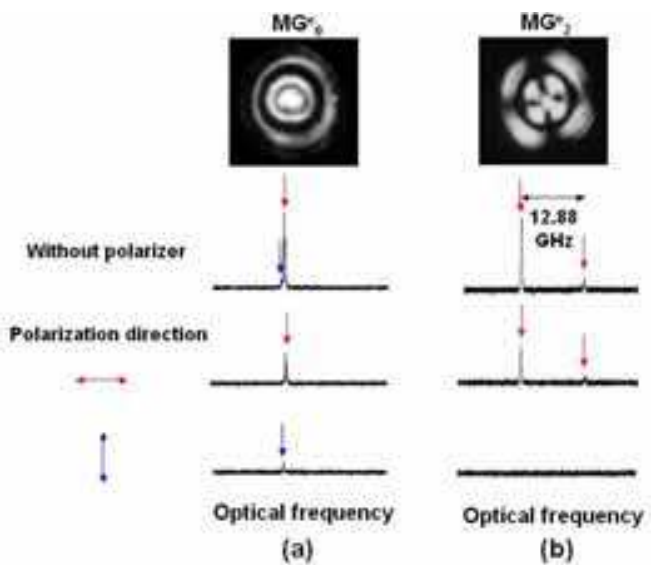

Fig. 20. Far-field lasing patterns and their polarization-dependent optical spectra.

(a) Dual-polarization Mathieu-Gauss beam with small off-axis pumping.

(b) Linear-polarization multi-longitudinal mode Mathieu-Gauss beam with large off-axis pumping.

elliptical coordinates were observed depending on the pump-beam position (Ohtomo et al., 2007), similar to large grain Nd:YAG ceramic lasers with spatially dependent thermal birefringence discussed in the previous subsection 4.1. Examples are shown in Fig. 21. As for large-grain Nd:YAG ceramic lasers, neither BG nor MG mode oscillations appeared with azimuth LD pumping.

(a)

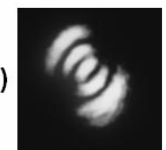
$\mathrm{IG}_{4,0}^{\mathrm{e}}$

(b)

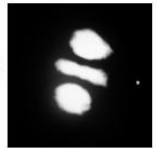

$\mathrm{IG}^{\mathrm{e}}{ }_{2,2}$

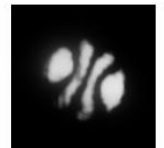

$\mathrm{IG}_{3,3}$

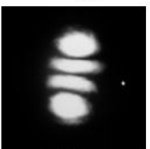

$\mathrm{IG}_{3,3}$

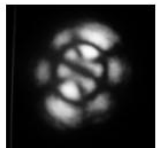

$\mathrm{IG}_{5,3}$

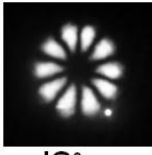

$\mathrm{IG}^{\mathrm{e}}{ }_{5,5}$

Fig. 21. Ince-Gauss mode operations with azimuth LD pumping. (a) $\mathrm{Nd}: \mathrm{GdVO}_{4}$ single crystal. (b) Large-grain Nd:YAG ceramic with average grain size of $19.2 \mu \mathrm{m}$.

\section{Discussion}

Laser oscillations in BG and MG modes are usually obtained in cavities with an axicon-type lens or mirror (Gutierrez-Vega, 2003; Alvarez-Elizondo, 2008) such that interference between conical lasing fields occurs within the laser cavity. In the present experiment, BG and MG mode oscillations were produced just by azimuth LD pumping. Let us offer a plausible explanation for MG mode oscillations in terms of effective off-axis pumping depicted in Fig. 22(a).

In the framework of vector lasers (Kravtsov, 2004), the angular amplification inhomogeneity has been shown to depend on the orientation of the polarization plane of laser radiation from that of pump radiation, in the form of $D(\theta, \Psi)=2 A_{0} \cos ^{2}(\theta-\Psi)$ as depicted in Fig. 22(b), 
and the polarization state is almost completely determined by the polarization of the pump radiation for an isotropic cavity with micro-grained Nd:YAG ceramic as described in section $\mathbf{3}$ (Ohtomo, 2007; Otsuka 2008). For azimuth LD pumping, the laser emission tends to occur such that its polarization direction follows the LD polarization direction within the pumped area.

Let us assume a small reflection loss difference at uncoated surfaces of the thermal lens between polarizations along radial and azimuth directions as depicted in Fig. 22(c). With the two effects combined, the laser polarization state may depend on the pump-beam position and size, i.e., gain area, if the LD polarization direction is fixed. For larger off-axis pumping, MG modes with a linear eigen-polarization are expected as a result of the stronger polarization discrimination effect and beam bending through the thermal lens as shown in Fig. 22(a). For small off-axis pumping, BG modes with orthogonal eigen-polarizations appear presumably because radial polarization components with a smaller reflection loss increase within the gain area.

(a)

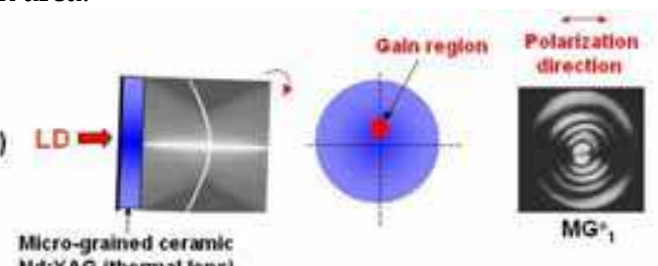

(b)

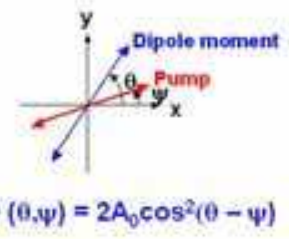

(c)

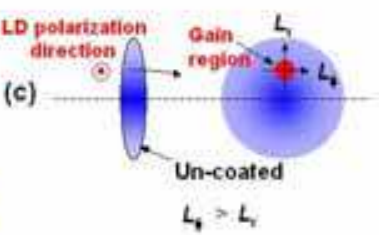

Fig. 22. (a) Conceptual illustration of the optical resonator containing a micro-grained Nd:YAG thermal lens with azimuth LD pumping. (b) Angle-dependent dipole moment induced by a linearly-polarized LD pump light. (c) Polarization-dependent reflection loss at un-coated surfaces.

In anisotropic lasers or large-grain Nd:YAG ceramic lasers, the laser polarization state is determined by fluorescence anisotropies or local thermal birefringence independently of the pump polarization, and neither BG nor MG mode oscillations take place.

\section{Concluding remarks}

In this Chapter, reviews were given on modal and polarization properties of microchip $\mathrm{Nd}$ :YAG ceramic lasers with laser-diode end pumping, featuring such effects as average grain sizes and azimuth pumping.

Segregations into multiple local-modes and the associated variety of dynamic instabilities occur in LD-pumped Nd:YAG samples with average grain size over several tens of microns resulting from the field interference effect among local-modes. The following results have been obtained for realizing stable single-frequency, linearly-polarized oscillations in Nd:YAG microchip ceramic lasers:

1. Micro-grained ceramics, whose average grain sizes are below $5 \mu \mathrm{m}$, can guarantee stable linearly-polarized TEM 00 mode operations. 
2. Large-grain ceramics, whose average grain sizes are larger than several tens of microns, can exhibit stable linearly-polarized oscillations in forced Ince-Gauss modes with azimuth/off-axis pumping.

3. Micro-grain ceramics can produce spontaneous Mathieu-Gauss and Bessel-Gauss lasing modes with azimuth/off-axis pumping.

\section{References}

Alvarez-Elizondo, M. B., Rodrlguez-Masegosa, R. \& Gutierrez-Vega, J. C. (2008). Generation of Mathieu-Gauss modes with an axicon-based laser resonator. Opt. Express 16, 23 (2008) 18770-18775, eISSN 1094-4087.

Arlt, J., Dholakia, K., Allen, L. \& Padgett, M. J. (1998). The production of multiringed Laguerre-Gaussian modes by computer-generated holograms. J. Mod. Opt. 45, 6 (1998) 1231-1237, ISSN 0950-0340.

Bandres, M. A. \& Gutierrez-Vega, J. C. (2004). Ince-Gaussian modes of the paraxial wave equation and stable resonators. J. Opt. Soc. Am. A 21, 5 (2004) 873-880, ISSN 1084-7529.

Bielawski, S., Derozier, D. \& Glorieux, P. (1992). Antiphase dynamics and polarization effects in the Nd-doped fiber laser. Phys. Rev. A 46, 5 (1992) 2811-2822, ISSN 1050-2947.

Cabrera, E., Calderon, O. G. \& Guerra, J. M. (2005). Experimental evidence of antiphase population dynamics in lasers. Phys. Rev. A 72 (2005) 043824, ISSN 1050-2947.

Chu, S.-C. \& Otsuka, K. (2007). Numerical study for selective excitation of Ince-Gaussian modes in end-pumped solid-state lasers. Optics Express 15 (2007) 16506-16519, eISSN 1094-4087.

Durin, J. (1987). Exact solutions for nondiffracting beams. I. The scalar theory. J. Opt. Soc. Am. A, 4, 4 (1987) 651-654, ISSN 1084-7529.

Erneux, T (1990). Laser Bifurcations, Northwestern University Press, Evanston, IL.

Gutierrez-Vega, J. C., Rodrlguez-Masegosa, R. \& Chaves-Cerda, S. (2003). Bessel-Gauss resonator with spherical output mirror: geometrical- and wave-optics analysis. J. Opt. Soc. Am. A 20, 11 (2003) 2113-2122, ISSN 1084-7529.

Ikesue, A., Furusato, I. \& Kamata, K. (1995a). Fabrication of polycrystalline, transparent YAG ceramics by a solid-state reaction method. J. Am. Ceram. Soc. 78, 1 (1995) 225228, ISSN 0002-7820.

Ikesue, A., Kinoshita, T., Kamata, K. \& Yoshida, K. (1995b). Fabrication and optical properties of high-performance polycrystalline Nd:YAG ceramics for solid-state lasers. J. Am. Ceram. Soc. 78, 4 (1995) 1033-1040, ISSN 0002-7820.

Kawai, R., Miyasaka, Y., Otsuka, K., Ohtomo, T., Narita, T., Ko, J.-Y., Shoji, I. \& Taira, T. (2004). Oscillation spectra and dynamic effects in a highly-doped microchip Nd:YAG ceramic laser. Opt. Express 12, 10 (2004) 2293-2302, eISSN 1094-4087.

Kimura, T. \& Otsuka, K. (1971). Thermal effects of a continuously pumped $\mathrm{Nd}^{3+}:$ YAG laser. IEEE J. Quantum Electron. QE-7, 8 (1971) 403-407, ISSN 00189197.

Ko, J.-Y., Otsuka, K. \& Kubota, T. (2001). Quantum-noise-induced order in lasers placed in chaotic oscillation by frequency-shifted feedback. Phys. Rev. Lett. 86, 18 (2001) 40254028, ISSN 0031-9007.

Koechner, W. \& Rice, D. K. (1970). Effect of birefringence on the performance of linearly polarized YAG:Nd lasers. IEEE J. Quantum Electron. QE-6,9 (1970) 557-566, ISSN 00189197.

Kravtsov, N. V., Lariontsev, E. G. \& Naumkin, N. I. (2004). Dependence of polarisation of radiation of a linear Nd:YAG laser on the pump radiation polarization. Quantum Electron. 34, 9 (2004) 839-842, ISSN 1063-7818. 
Lu, J., Prabhu, M., Xu, J., Ueda, K., Yagi, H., Yanagitani, T. \& Kaminskii, A. (2000). Highly efficient 2\% Nd:yttrium aluminum garnet ceramic laser. Appl. Phys. Lett. 77, 23 (2000) 3707-3709, ISSN 0003-6951.

Narita, T., Miyasaka, Y. \& Otsuka, K. (2005). Self-Induced instabilities in $\mathrm{Nd}_{2} \mathrm{Y}_{3} \mathrm{Al}_{5} \mathrm{O}_{12}$ ceramic lasers. Jpn. J. Appl. Phys. 37 (2005) L1168-L1170, ISSN 0021-4922.

Ohtomo, T., Kamikariya, K. \& Otsuka, K. (2007). Effect of grain size on modal structure and polarization properties of laser-diode-pumped miniature ceramic lasers. Jpn. J. Appl. Phys. 46 (2007) L1043-L1045, ISSN 0021-4922.

Ohtomo, T., Kamikariya, K., Otsuka, K. \& Chu, S.-C. (2007). Single-frequency Ince-Gaussian mode operations of laser-diode-pumped microchip solid-state lasers. Opt. Express 15, 17 (2007) 10705-10717, eISSN 1094-4087.

Ohtomo, T. \& Otsuka, K. (2009). $\mathrm{Yb}_{3} \mathrm{Y}_{3} \mathrm{Al}_{5} \mathrm{O}_{12}$ laser for self-mixing laser metrology with enhanced optical sensitivity. Jpn. J. Appl. Phys. 48 (2009) 070212, ISSN 0021-4922.

Otsuka, K. (1999). Nonlinear Dynamics in Optical Complex Systems. Kluwer Academic Publishers. Dordrecht/London/Boston (1999), Chapter 2, ISBN 07923-6132-6.

Otsuka, K., Kawai, R., Hwong, S.-L., Ko, J.-Y. \& Chern, J.-L. (2000). Synchronization of mutually coupled self-mixing modulated lasers. Phys. Rev. Lett. 84, 14 (2000) 30493052, ISSN 0031-9007.

Otsuka, K., Ko, J.-Y., Lim, T.-S., and Makino, H. (2002). Modal interference and dynamical instability in a solid-state slice laser with asymmetric end-pumping. Phys. Rev. Lett. 87 (2002) 083903, ISSN 0003-6951.

Otsuka, K., Narita, T., Miyasaka, Y., Ching, C.-C., Ko, J.-Y. \& Chu, S.-C. (2006). Nonlinear dynamics in thin-slice Nd:YAG ceramic lasers: Coupled local-mode model. Appl. Phys. Lett. 89, 8 (2006) 081117, ISSN 0003-6951.

Otsuka, K., Nemoto, K., Kamikariya, K., Miyasaka, Y., Ko, J.-Y. \& Lin, C.-C. (2007). Chaos synchronization among orthogonally polarized emissions in a dual-polarization laser. Phys. Rev. E 76, 2 (2007) 026204, ISSN 1063-651X.

Otsuka, K., Nemoto, K., Kamikariya, K., Miyasaka, Y. \& Chu, S.-C. (2007). Linearly polarized single-frequency oscillations of laser-diode-pumped microchip ceramic Nd:YAG lasers with forced Ince-Gaussian mode operations. Jpn. J. Appl. Phys. 46 (2007) 58655867, ISSN 0021-4922.

Otsuka, K. \& Ohtomo, T. (2008). Polarization properties of laser-diode-pumped micro-grained Nd:YAG ceramic lasers. Laser Phys. Lett. 5, 9 (2008) 659-663, ISSN 1612-2011.

Schwarz, U. T., Bandres, M. A. \& Gutierrez-Vega, J. C. (2004). Observation of Ince-Gaussian modes in stable resonators. Opt. Lett. 29, 16 (2004) 1870-1872, ISSN 0146-9592.

Shoji, I., Kurimura, S., Sato, Y., Taira, T., Ikesue, A. \& Yoshida, K. (2000). Optical properties and laser characteristics of highly Nd3 + -doped Y3Al5O12 ceramics. Appl. Phys. Lett. 77, 7 (2000) 939-941, ISSN 0003-6951.

Shoji, I., Sato, Y., Kurimura, S., Lupei, V., Taira, T., Ikesue, A. \& Yoshida, K. (2002). Thermalbirefringence-induced depolarization in Nd:YAG ceramics. Opt. Lett. 27, 4 (2002) 234-236, ISSN 0146-9592.

Sudo, S., Miyasaka, Y., Kamikariya, K., Nemoto, K. \& Otsuka, K. (2006). Microanalysis of Brownian particles and real-time nanometer vibrometry with a laser-diodepumped self-mixing thin-slice solid-state laser. Jpn. J. Appl. Phys. 45 (2006) L926L928, ISSN 0021-4922.

Tokunaga, K., Chu, S.-C., Hsiao, H.-Y., Ohtomo, T. \& Otsuka, K. (2009). Spontaneous Mathieu-Gauss mode oscillation in micro-grained Nd:YAG ceramic lasers with azimuth laser-diode pumping. Laser Phys. Lett. 6, 9 (2009) 635-638, ISSN 1612-2011. 


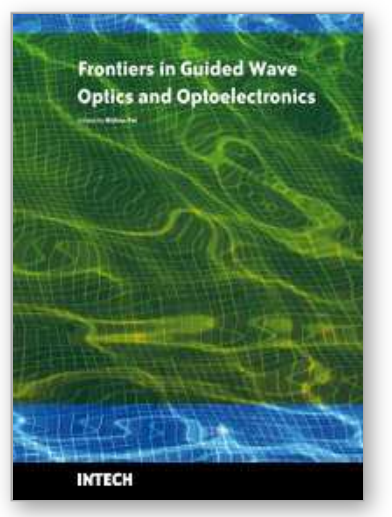

\author{
Frontiers in Guided Wave Optics and Optoelectronics \\ Edited by Bishnu Pal
}

ISBN 978-953-7619-82-4

Hard cover, 674 pages

Publisher InTech

Published online 01, February, 2010

Published in print edition February, 2010

As the editor, I feel extremely happy to present to the readers such a rich collection of chapters authored/coauthored by a large number of experts from around the world covering the broad field of guided wave optics and optoelectronics. Most of the chapters are state-of-the-art on respective topics or areas that are emerging. Several authors narrated technological challenges in a lucid manner, which was possible because of individual expertise of the authors in their own subject specialties. I have no doubt that this book will be useful to graduate students, teachers, researchers, and practicing engineers and technologists and that they would love to have it on their book shelves for ready reference at any time.

\title{
How to reference
}

In order to correctly reference this scholarly work, feel free to copy and paste the following:

Kenju Otsuka (2010). Polarization Properties of Laser-Diode-Pumped Microchip Nd:YAG Ceramic Lasers, Frontiers in Guided Wave Optics and Optoelectronics, Bishnu Pal (Ed.), ISBN: 978-953-7619-82-4, InTech, Available from: http://www.intechopen.com/books/frontiers-in-guided-wave-optics-andoptoelectronics/polarization-properties-of-laser-diode-pumped-microchip-nd-yag-ceramic-lasers

\section{INTECH}

open science | open minds

\section{InTech Europe}

University Campus STeP Ri

Slavka Krautzeka 83/A

51000 Rijeka, Croatia

Phone: +385 (51) 770447

Fax: +385 (51) 686166

www.intechopen.com

\section{InTech China}

Unit 405, Office Block, Hotel Equatorial Shanghai

No.65, Yan An Road (West), Shanghai, 200040, China

中国上海市延安西路65号上海国际贵都大饭店办公楼405单元

Phone: +86-21-62489820

Fax: +86-21-62489821 
(C) 2010 The Author(s). Licensee IntechOpen. This chapter is distributed under the terms of the Creative Commons Attribution-NonCommercialShareAlike-3.0 License, which permits use, distribution and reproduction for non-commercial purposes, provided the original is properly cited and derivative works building on this content are distributed under the same license. 